\title{
A Novel Compression Algorithm Based on Bio-Orthogonal Transform and Encoding Methods for Computed Tomography Images
}

\author{
R.Pandian ${ }^{\mathrm{a}, 1}$, S LalithaKumari ${ }^{\mathrm{b}}$, V.AmalaRani ${ }^{\mathrm{b}}$, B.PrabhuDassBatvari ${ }^{\mathrm{b}}$ and \\ R. RajaKumar \\ ${ }^{\mathrm{a}, 1, \mathrm{~b}}$ Associate Professor, Sathyabama Institute of Science and Technology, Chennai, \\ Tamil Nadu \\ ${ }^{c}$ Professor, Sathyabama Institute of Science and Technology, Chennai
}

\begin{abstract}
Image compression is the processing of images by using Transform operations and Encoding techniques. Nowadays, there is an essential need of these methods in the medical field. This work is focused on the performance quality assessment of medical images using Image Compression Techniques. Image compression is the process of compression of an image in such a way that it has less space than the original image. It is an organization of compression technique that reduces the size of an image file without affecting its quality to a greater extent. The bio-orthogonal transform is used for decomposing the Lung images. After decomposition, different methods of encoding are performed and finally the proposed compression methods are evaluated for finding optimum algorithm for medical image.
\end{abstract}

Keywords: Wavelet, Encoding, FT, FFT, SPIHT, EZW and STW.

\section{Introduction}

Image processing assumes a significant part in the fields like medicine, machine vision and example acknowledgment. The each piece of data is an image that is required in future for performing errands like arrangement, recovery and acknowledgment. The image compression is important to keep transmission capacity and save memory space. The computerized images are frequently characterized into varying sorts e.g double, dim scale, shading, bogus - shading, multispectral and thematic[1],[2].The change configuration might be a refinement of the two-sided separating strategy which contemplates the likeliness of shading pixels and their fleeting reach. The middle channel is very proficient in eliminating

${ }^{1}$ Pandian R, Associate professor, Dept of ECE, Sathyabama Institute of Science and Technology

Email:rpandianme@gmail.com 
Imprudent commotion, but its presentation is reduced when the image is slanted by Gaussian clamor and henceforth the VMF is normally matched with other separating arrangements in such a circumstance. This methodology focuses on a semi-nearby normal of the image pixels in such a way that the images can be safeguarded. The new pixel esteem is estimated as a weighted normal of the pixels which particular district is near the precarious edge of the pixel's nearby area that is being dissected. Regardless, the conventional channel applications normally obscure level inclinations inside the outcomes which can adversely influence ensuing transformations that mutilate the exact lines of the correlative constructions. The restriction of the above customary de-noising approaches frequently takes place in climate processing. To disentangle this issue, a few seismologists have followed the kind of edge-saving strategy which used in advanced image investigation.

It is shown from different trials that such a framework can productively eliminate the clamor while holding solid inclinations. According to literature overview, this work is engaged to address the compression algorithms by bio-symmetrical transform. The paper is organized as follows. In section 2 discusses how arrangements are done with the Image data base and section 3 clearly explains about the wavelet Transform. The results are discussed in section 4 and the exploration work is concluded in section 5 .

\section{Image Database}

The prepared computed tomography of a lung considered in this work resembles the developed x-radiates using a couple of pictures or photographs that shows the internal organs of the human. The standard lung pictures are taken into account for depicting the threats in lung. An illustration of common place and threatening development affected CT pictures are shown in Figure 1. The pictures used for testing the estimations made are clinical pictures. In this work, medical images like the normal CT lung pictures and a threat impacted CT lung pictures taken from ten persons of a social class in DICOM design are used to depict the lung infection. The image data for this investigation work is amassed as conventional and strange pictures. The typical lung pictures are shown in Figure 1. The CT Lung pictures are gathered from Sathyabama Institute of science and Technology hospital, Chennai, TamilNadu.

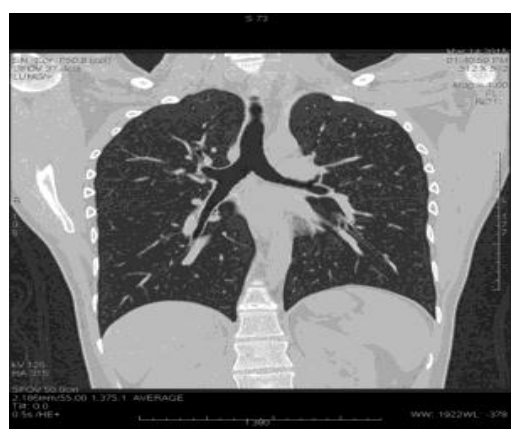

Figure 1.Normal Lung coronal view 


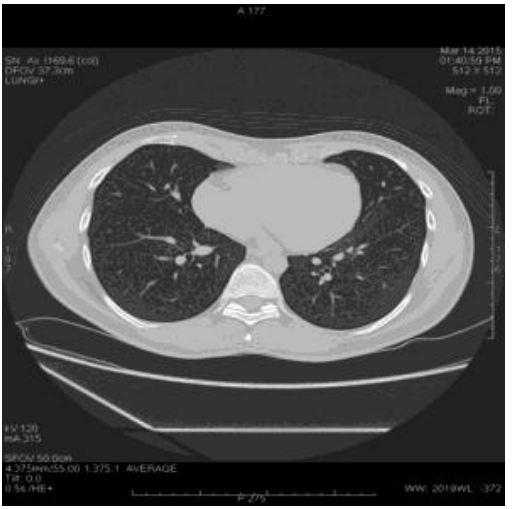

Figure 2.Normal Lung axial view

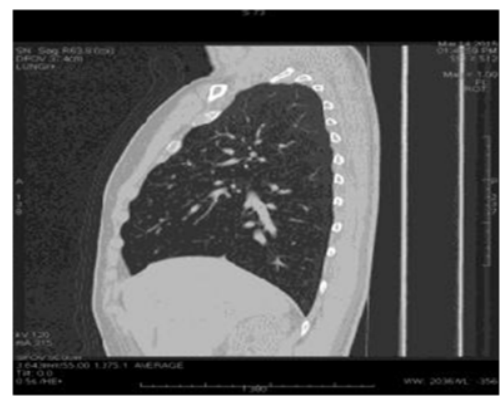

Figure 3. Normal Lung sagittal view

\section{Wavelet Transforms}

In Compression, it should be underlined that Fourier change includes averaging of the sign with a period direction that brings a few misfortunes inside the nitty gritty transient data of the sign. The Fourier change also includes a hard and fast goal for all frequencies. Conversely, the wavelet examination changes a picture inside the time area into a recurrence space with various goals at various sign frequencies. It gives a multi-goal thanks to the effect of image investigation.[3] The wavelet approach gets a period scale deterioration of the sign under consideration utilizing an interpretation (time) boundary and a scale boundary. There are two methodologies namely persistent wavelet changes (CWT) and discrete wavelet changes (DWT). In both CWT and DWT approaches, the understanding boundary is discrete, though the dimensions boundary is permitted to fluctuate consistently in CWT, [4] yet it is discrete in DWT. So, on beat impediments in pressure, a couple of methodologies are proposed to upheld time-recurrence confinement almost like envelope examination, Gabor Windowed Fourier Transform (GWFT) and wavelet investigation techniques. Generally, [4] the Fourier change (FT) is broadly utilized in image handling. It does not give time confinement. So, it is seldom suitable for non-fixed cycles. Along these lines, it is less valuable in breaking down non-fixed information where there is no reiteration inside the district sampled[5]. Furthermore, one of the restrictions of Fast Fourier Transform 
(FFT) in image investigation is that the nonappearance of worldly data. The brief time frame that the Fourier changes (STFT) confines time by moving time window. However, the fixed width is kept as far as possible at the high- recurrence run. The wavelet changes permit the segments of a non-fixed sign to be investigated, permit channels to be built for both fixed and non-fixed signals and have a window whose transmission capacity shifts in reference to the recurrence of the wavelet.[5]. It clarifies a full decay of the image was done to measure wavelet coefficients was an equal on the grounds that of the primary image and can be recombined to recreate the most image. It is accepted that wavelet investigation can assume a serious part in pressure research for diagnostics device.[6]. The Wavelet Transform is a broadly received strategy for pressure. The Essential pressure plot during this strategy is actualized inside the accompanying request relationship, quantization and encoding. DCT and DWT are documented, the changes are used to relate the pixels. The wavelet change deteriorates the image into various recurrence sub groups, to be specific lower recurrence sub groups and better recurrence sub groups by which smooth varieties and subtleties of the image are frequently isolated. The bulk of the energy is compacted into lower recurrence subgroups[7].The greater part of the coefficients in higher recurrence sub groups are little or zero and have a twisted to be assembled and furthermore are situated inside an identical relative spatial inside the sub groups. Hence, pressure strategies utilize wavelet changes which are effective in giving high paces of pressure while maintaining great image quality and are better than DCT-based techniques. In DCT, an outsized portion of the energy is compacted into lower recurrence coefficients to quantization, the overwhelming majority of the upper recurrence coefficient become little or zero and have a twisted to be assembled.[8]

The LL sub bands are frequently additionally disintegrated for ensuing degree of decay. On the off chance, that the degree of disintegration expands, the higher subtleties are caught more effectively [9]. The image subtleties are pressed into a little number of coefficients which are decreased to less number by following a quantization. The blunder or misfortune in data is on account of the quantization step. In this work, bio-orthogonal is used for decomposing the lung images for various vanishing moments. The performances are measured by vanishing moments for different encoder like SPIHT, EZW an STW [10] [11].

\section{Results and Discussions}

The decomposition levels and vanishing moments are analyzed based on MSE, PSNR compression ratio and Bits per pixel. From the Table.1, the quality of compression evaluated by PSNR and amount of compression measured by MSE. The performance of compression using Biorthogonal Transform with five encoding methods for various vanishing moments such as 1.1,1.3,1.5.1.7and 1.9 with various decomposition levels are shown in Table 1 
Table 1. Performance evaluation of Compression Algorithms

\begin{tabular}{|c|c|c|c|c|c|}
\hline Transform Type & Encod & PSNR & CR(\%) & BPP & MSE \\
\hline \multirow{3}{*}{ Bior 1.3 with level1 } & SPIHT & 42.14 & 67.02 & 17.05 & 5.01 \\
\hline & EZW & 86.02 & 89.2 & 25.02 & 0.03 \\
\hline & STW & 61.02 & 86.06 & 21.08 & 0.02 \\
\hline \multirow{3}{*}{ Bior 1.3 with level 2} & SPIHT & 41.54 & 66.07 & 15.85 & 4.56 \\
\hline & EZW & 64.57 & 88.70 & 21.28 & 0.02 \\
\hline & STW & 55.23 & 85 & 20.39 & 0.19 \\
\hline \multirow{3}{*}{ Bior 1.3 with level 3} & SPIHT & 40.35 & 39.58 & 9.49 & 6 \\
\hline & EZW & 52.10 & 76.09 & 18.26 & 0.41 \\
\hline & STW & 46.08 & 59.08 & 14.18 & 1.61 \\
\hline \multirow{3}{*}{ Bior 1.3 with level 4} & SPIHT & 36.41 & 20.97 & 5.03 & 14.87 \\
\hline & EZW & 44.79 & 51.85 & 12.44 & 2.16 \\
\hline & STW & 38.42 & 31.23 & 7.5 & 9.4 \\
\hline \multirow{3}{*}{ Bior 1.5 with level1 } & SPIHT & 41.02 & 66.58 & 16.81 & 5.58 \\
\hline & EZW & 82.9 & 89.12 & 23.8 & 0.4 \\
\hline & STW & 60.08 & 85.78 & 22.02 & 0.02 \\
\hline \multirow[t]{3}{*}{ Bior 1.5 with level 2} & SPIHT & 40.75 & 66.31 & 15.91 & 5.47 \\
\hline & EZW & 63.83 & 89.48 & 21.48 & 0.03 \\
\hline & STW & 54.85 & 85.56 & 20.53 & 0.21 \\
\hline \multirow[t]{5}{*}{ Bior 1.5 with level3 } & EZW & 43.62 & 52.53 & 11.94 & 1.97 \\
\hline & STW & 46.14 & 60.2 & 15.28 & 2.01 \\
\hline & SPIHT & 37.01 & 22.12 & 6.01 & 13.58 \\
\hline & EZW & 37.57 & 29.80 & 7.01 & 9.56 \\
\hline & STW & 39.15 & 33.32 & 7.26 & 9.12 \\
\hline
\end{tabular}




\section{Conclusion}

A high amount of compression is achieved by Bio-orthogonal Transform with Encoding methods. The original image and compressed images are evaluated pixel by pixel. It is found that the compressed image will give more information. If the number of vanishing moments are increased, the quality of image is almost same. The bpp of the proposed calculations are additionally practically of same worth. It is apparent from the outcomes that the idea of the mother wavelets is the reason for the adjustment in the presentation esteems. The results obviously uncover that the quantity of evaporating minutes likewise assumes a significant part in the pressure calculation. It is likewise obvious that the ascent in the quantity of evaporating minutes prompts misfortune in data.

\section{Acknowledgment}

The authors are grateful to The Management of Sathyabama Institute of Science Technology, Chennai, TamilNadu, India.

\section{References}

[1] Pandian R,Vigneswaran T \&LalithaKumari S, Characterization of CT cancer lung image using image compression algorithms and feature Extraction, Journal of Scientific and Industrial Research , 75 (2016),pp747-751.

[2] Syed Mortadza SA, Sim JA, Stacey M, Jiang LH. Signalling mechanisms mediating Zn(2+)-induced TRPM2 channel activation and cell death in microglial cells, "Scientific Repots ,Vol.7,2017

[3] R Stephen, K Hongisto, A Solomon, Physical Activity and Alzheimer's Disease: A Systematic Review, The Journals of Gerontology: Series A, Vol. 72(6), pp. 733-7, 2017.

[4] Tijn M. Schouten Anne Hafkemeijer Elise G. P Combining anatomical MRI measures improves Alzheimer's disease classification ,National Library of medicine, Vol .35(5),2016,pp. 1920-1929.

[5] Tijn M. Schouten Anne Hafkemeijer Elise G. P .Combining anatomical MRI measures improves Alzheimer's disease classification ,Neuro Image Clinic. Vol .35(5),2016,pp. 1920-1929.

[6] Sheela K G \&Deepa S N, Selection of number of hidden neurons in renewable energy system, Journal of Scientific and Industrial Research, $173(2014), 686-688$

[8] Pandian R, Evaluation of image compression algorithms, IEEE Und Tech (UT) NIOT, (2015) 1-3

[9] Pandian R \&Vigneswaran T, Adaptive wavelet packet basis selection for zerotree image coding, International Journal of Signal and Imaging Systems Engineering, 9 (2016)388-392.

[10] Pandian R .Development of Algorithms for Medical Image Compression: Compression Algorithms, Examining Fractal Image Processing and Analysis.2020.

[11] V. D. AK, S. Sharmila, Abhishek Kumar, A. K. Bashir, Mamoon Rashid, Sachin Kumar Gupta \&Waleed S. Alnumay . novel solution for finding postpartum haemorrhage using fuzzy neural techniques.Neural Computing and Applications (2021) (https://doi.org/10.1007/s00521-020-05683-z)

[12] R. Subha Shini et.al., " Recurrent Neural Network based Text Summarization Techniques by Word Sequence Generation”,IEEE International Conference on Inventive Computation Technologies (ICICT), 2021, DOI: 10.1109/ICICT50816.2021.9358764 